\title{
Correction to: Message Exchange Games in Strategic Contexts
}

\author{
Nicholas Asher ${ }^{1}$ - Soumya Paul ${ }^{2}$. \\ Antoine Venant ${ }^{2}$
}

\section{Correction to: J Philos Logic (2017) 46:355-404 https://doi.org/10.1007/s10992-016-9402-1}

Our paper, 'Message Exchange Games in Strategic Contexts' lost pertinent information about funding and acknowledgments on its way to publication. We thus include them in this erratum here.

We thank Julie Hunter, Alex Lascarides, David Beaver, Eric McCready, Daisuke Bekki, Chris Barker, Erich Grädel, Hans Kamp, Benedikt Löwe, Julian Schlöder, Itai Sher, to the participants of the Rutgers Workshop on Coordination and Content and to an anonymous reviewer for the Journal of Philosophical Logic for their helpful comments on previous versions of this paper.

This work was supported by ERC grant 269427.

The online version of the original article can be found at https://doi.org/10.1007/s10992-016-9402-1.

Nicholas Asher

nicholas.asher@irit.fr

1 CNRS, IRIT, Toulouse, France

2 Université de Toulouse 3, IRIT, Toulouse, France 